Meta

Journal des traducteurs

Translators' Journal

\title{
La terminologie de l'alpinisme dans les dictionnaires
}

\section{Christiane Tetet}

Volume 39, numéro 4, décembre 1994

Hommage à Bernard Quemada : termes et textes

URI : https://id.erudit.org/iderudit/004467ar

DOI : https://doi.org/10.7202/004467ar

Aller au sommaire du numéro

\section{Éditeur(s)}

Les Presses de l'Université de Montréal

\section{ISSN}

0026-0452 (imprimé)

1492-1421 (numérique)

Découvrir la revue

Citer cet article

Tetet, C. (1994). La terminologie de l'alpinisme dans les dictionnaires. Meta, 39(4), 651-661. https://doi.org/10.7202/004467ar

\section{Résumé de l'article}

L'alpinisme a connu, depuis ses origines, un développement remarquable : extension de son "terrain de jeu", diversification, spécialisation, modification dans sa pratique avec l'utilisation de nouvelles techniques, de nouveaux équipements. L'escalade, moyen d'accès à l'alpinisme, devient aujourd'hui discipline indépendante lorsqu'elle s'exerce en falaises, sur blocs et sur structures artificielles. Nous étudierons dans cet article comment ce vocabulaire de spécialité a été appréhendé par les auteurs de dictionnaires de langue généraux et de dictionnaires spécialisés monolingues et multilingues en nous limitant à deux aspects du traitement lexicographique : l'analyse des nomenclatures alpines et l'information historique, cette dernière n'ayant pas, à notre connaissance, encore été envisagée par les métalexicographes pour ce qui concerne les vocabulaires de spécialités. L'élaboration de nomenclatures actualisées, représentatives du nouvel alpinisme, devrait permettre la mise en chantier d'un répertoire multilingue indispensable à la pratique, dans le cadre de l'adaptation à une nouvelle politique économique du milieu de la montagne. Notre analyse de la nature des informations historiques proposées dans les dictionnaires de langue, à travers la mention des premières attestations, fera apparaître la confusion existant actuellement, entre datation-diffusion et datation-création, lorsque la datation est affaire des lexicographes. Les travaux dans ce domaine, entrepris à l'initiative du professeur Quemada, et menés au sein de l'équipe Matériaux pour l'histoire du vocabulaire français de l'INaLF, vont dans ce sens.
Tous droits réservés (C) Les Presses de l'Université de Montréal, 1994
Ce document est protégé par la loi sur le droit d'auteur. L’utilisation des services d'Érudit (y compris la reproduction) est assujettie à sa politique d'utilisation que vous pouvez consulter en ligne.

https://apropos.erudit.org/fr/usagers/politique-dutilisation/ 


\title{
LA TERMINOLOGIE DE L'ALPINISME DANS LES DICTIONNAIRES
}

Christiane TETET

INaLF-CNRS, Besançon, France

\begin{abstract}
Résumé
L'alpinisme a connu, depuis ses origines, un développement remarquable: extension de son "terrain de jeu», diversification, spécialisation, modification dans sa pratique avec l'utilisation de nouvelles techniques, de nouveaux équipements. L'escalade, moyen d'accès à l'alpinisme, devient aujourd' hui discipline indépendante lorsqu' elle s'exerce en falaises, sur blocs et sur structures artificielles.

Nous étudierons dans cet article comment ce vocabulaire de spécialité a été appréhendé par les auteurs de dictionnaires de langue généraux et de dictionnaires spécialisés monolingues et multilingues en nous limitant à deux aspects du traitement lexicographique: l'analyse des nomenclatures alpines et l' information historique, cette dernière n'ayant pas, $\grave{a}$ notre connaissance, encore été envisagée par les métalexicographes pour ce qui concerne les vocabulaires de spécialités.

L'élaboration de nomenclatures actualisées, représentatives du nouvel alpinisme, devrait permettre la mise en chantier d'un répertoire multilingue indispensable à lá pratique, dans le cadre de l'adaptation à une nouvelle politique économique du milieu de la montagne.

Notre analyse de la nature des informations historiques proposées dans les dictionnaires de langue, à travers la mention des premières attestations, fera apparaitre la confusion existant actuellement, entre datation-diffusion et datation-création, lorsque la datation est affaire des lexicographes. Les travaux dans ce domaine, entrepris à l'initiative du professeur Quemada, et menés au sein de l'équipe Matériaux pour l'histoire du vocabulaire français de l'INaLF, vont dans ce sens.
\end{abstract}

\begin{abstract}
Since its origin, mountaineering has undergone exceptional development: extension of its "playground", its diversification, specialization and practice that has been modified by the use of new techniques and equipment. Rockclimbing, once an introduction to mountaineering, has now become an independent sport in its own right when practised on cliffs, blocks and artificial structures.

This paper describes how this specialized vocabulary has been dealt with by the authors of both general and monolingual as well as multilingual specialized dictionaries. Two aspects of the lexicographical treatment of this vocabulary are investigated: firstly an analysis of mountaineering nomenclature and secondly its historical background which, to the best of our knowledge, has not been taken into account by metalexicographers for specialized vocabularies.

The compilation of updated nomenclatures, representative of current mountaineering practice, would lead to the multilingual glossary that is needed for this sport within a framework of a new economic policy for mountaineering.

An analysis of the historical data, i.e. first attestations, in language dictionaries demonstrates that there is confusion among lexicographers concerning the creation date of $a$ term and its wider distribution date. On the initiative of Professor Quemada, work on dating is being carried out by the Materiaux pour l'histoire du vocabulaire français team of the INALF (CNRS).
\end{abstract}


L'étude critique du traitement des vocabulaires de spécialités dans les dictionnaires n'est pas récente. Elle s'est développée il y a une vingtaine d'années et continue de faire régulièrement l'objet d'articles dans les revues de linguistique ${ }^{1}$.

Dans tous les cas, le domaine de spécialité traité relève des sciences et des techniques. Et dans tous les cas, l'étude du traitement lexicographique de ces domaines s'est limité à l'analyse de la nomenclature et à la nature des définitions proposées, le plus souvent dans les dictionnaires de langue, monolingues, généraux.

Nous aborderons le problème ici en prenant en compte une discipline sportive les vocabulaires sportifs sont trop souvent les parents pauvres de la lexicologie et de la lexicographie - dans différentes réalisations lexicographiques, dont le texte multilingue.

Nous avons retenu comme discipline sportive l'alpinisme, sport qui se pratique en haute montagne, et sa composante escalade, à la fois discipline sportive complémentaire de l'alpinisme et discipline indépendante.

Nous avons choisi de traiter de l'alpinisme pour deux raisons : l'une, qui relève de la nature métalexicographique de cette étude: il ne nous apparaît pas possible d'aborder le vocabulaire d'un domaine sans connaître parfaitement ce domaine ou pratiquer la discipline et c'est notre cas - un point de vue que les lexicographes qui sont dans l'obligation d'aborder tous les domaines de la connaissance, contesteront -; l'autre raison tient à la discipline elle-même: il nous a paru intéressant d'analyser la représentation lexicographique d'une discipline qui n'a cessé d'évoluer et de se diversifier depuis ses origines jusqu'à nos jours.

Après avoir présenté un panorama des nomenclatures alpines - la seule élaboration d'une nomenclature est déjà significative du traitement qui sera dévolu au vocabulaire traité - nous nous limiterons dans cet article à l'analyse des informations historiques proposées dans les dictionnaires de langue lorsqu'ils traitent des vocabulaires spécialisés, un aspect, qui, à notre connaissance, est souvent négligé par les métalexicographes ${ }^{2}$.

Nous ne sous-estimons pas les difficultés que rencontre le dictionnariste dans le traitement des vocabulaires de spécialités. Mais nous pensons que ces difficultés pourraient être réduites, et par conséquent le traitement lexicographique mieux adapté, si le lexicographe se donnait les moyens d'aborder les domaines de spécialités avec plus de rigueur.

Nous ne nous limiterons pas dans cet article à une analyse critique, toujours facile, de l'existant; nous proposerons quelques pistes méthodologiques qui devraient permettre de mieux assurer le traitement lexicographique des vocabulaires de spécialités.

\section{L'ALPINISME D'HIER À AUJOURD'HUI}

L'intérêt pour la haute montagne se développe en Europe vers le milieu du XVIII siècle. Il est le fait de voyageurs, de savants, qui explorent le monde alpin à des fins scientifiques. Lorsqu'en 1786, Balmat et Paccard atteignent pour la première fois le sommet du Mont Blanc, c'est à la demande de Horace Benedict de Saussure, savant naturaliste, qui en accomplira la troisième ascension en 1787. À partir de ce moment l'exploration de la haute montagne s'intensifie; les visées scientifiques ne sont plus que prétextes à «excursions»; les ascensionnistes deviennent alors des sportifs. De 1850 à 1865 , les principaux sommets des Alpes sont atteints; puis ces mêmes sommets seront atteints par des voies différentes, plus difficiles.

Dès le début du XX $\mathrm{X}^{\mathrm{e}}$ siècle, les alpinistes se tournent vers l'Himalaya. De nombreuses tentatives ont lieu à l'Everest. Finalement, c'est en 1950 que le premier 8000 est atteint par une expédition française dirigée par Maurice Herzog. La conquête de l'Annapurna connaîtra un grand retentissement, non seulement dans le monde alpin, mais aussi auprès du grand public. En 1953, c'est la victoire au sommet de l'Everest.

En 1970, tous les sommets de plus de 8000 mètres sont conquis par les voies normales. 
Parallèlement, l'alpinisme prend des formes nouvelles : aux ascensions en été succèdent les ascensions hivernales; aux ascensions en équipe, les ascensions en solitaire. À partir de 1980 apparait l'alpinisme-express : atteindre un sommet dans un minimum de temps; puis plusieurs sommets sans temps de repos: les enchaînements en solitaire se multiplient, fortement médiatisés.

Alors que l'alpinisme apparaît comme une activité relativement ancienne, il n'a pas connu, auprès du grand public, le succès rencontré par d'autres sports apparus beaucoup plus tardivement (le football en 1872; le cyclisme au milieu du XIX ${ }^{\mathrm{e}}$ siècle; le ski au début du $\mathrm{XX}^{\mathrm{e}}$ siècle), en raison de la nature même de sa pratique: l'alpinisme, qui ne pouvait être un sport-spectacle, excluait la compétition 3 .

Aujourd'hui l'alpinisme est devenu un sport-spectacle par le biais des images télévisées; le nombre de ses pratiquants ne cesse d'augmenter. On compte environ 200000 alpinistes parmi les 5 millions de Français qui fréquentent la montagne; quant aux professionnels de l'alpinisme, les guides de haute montagne, ils sont entre 800 et 850 à exercer leur activité au sein d'associations ou auprès de particuliers ${ }^{4}$.

L'évolution qu'a connue l'alpinisme est indissociable de l'évolution que connât la pratique de l'escalade, considérée pendant longtemps comme un moyen d'accès à l'alpinisme et considérée toujours comme telle par les lexicographes: «escalade. ALP. Ascension (d'un sommet, d'une montagne) au cours de laquelle le grimpeur progresse en utilisant uniquement les prises et les appuis qu'offre le rocher [...] ou en utilisant des points d'appuis, pitons notamment, dans le rocher.» (Petit Larousse illustré 1993.)

Or, dans les années 80 , l'escalade, tout en restant une modalité d'accès à l'alpinisme, est devenue discipline sportive à part entière. L'escalade s'exerce maintenant hors du milieu haute montagne, soit sur sites naturels : falaises ou blocs — on recense plus de 1500 sites naturels en France ${ }^{5}$ - soit sur structures artificielles. Ainsi, après s'être exclue de l'environnement montagne, l'escalade s'est exclue de l'environnement rocheux. On comptait en 1989, en France, plus de 500 structures artificielles. L'éthique de l'escalade s'en est trouvée totalement modifiée: la compétition a fait son apparition dans un milieu qui l'avait ignorée jusqu'alors: compétition de vitesse ou de difficulté, au niveau régional, national, international. Et l'escalade est en passe de devenir discipline olympique.

Cette forme d'escalade, dite sportive, est reconnue depuis peu par les lexicographes qui l'enregistrent sous le terme grimpe; Le Petit Larousse, pour la première fois, en 1989 et Le Petit Robert dans son édition de 1993, qui le glose ainsi : «Escalade à mains nues de parois abruptes naturelles ou non.»6

Tout alpiniste est donc grimpeur; tout grimpeur n'est pas alpiniste. Le nombre de grimpeurs est relativement difficile à chiffrer. Ph. Bourdeau estime à 84000 le nombre de grimpeurs réguliers en 19857. Au $1^{\text {er }}$ janvier 1993, 206 grimpeurs possédaient le Brevet d'État de Monitorat d'escalade. L'escalade s'est professionnalisée en s'autonomisant.

Il n'est plus possible aujourd'hui en lexicographie, d'ignorer l'évolution de ces deux disciplines à la fois complémentaires et indépendantes, que sont l'alpinisme et l'escalade.

\section{LES NOMENCLATURES ALPINES}

Dans le cadre de recherches de nature historique sur le vocabulaire de l'alpinisme - recherche de la première attestation d'un terme - nous avons été amenée à recenser l'apparition d'environ 5000 termes entre le milieu du XVIII' siècle et $1990^{8}$. Nous estimons à environ 1500 le nombre de termes effectivement en usage aujourd'hui ${ }^{9}$.

Alors que l'alpinisme apparaît comme une discipline sportive dès le début du XIXe siècle, le mot est absent du Grand dictionnaire universel du XIX ${ }^{e}$ siècle de Pierre Larousse, qui, par ailleurs, ignore tout de l'activité qui consiste à escalader les montagnes. Il faut 
attendre 1898 pour que le mot apparaisse dans un répertoire lexicographique: «Passion pour la montagne, goût des excursions dans la montagne, et, d'une façon générale, tout ce qui se rapporte à ce genre de sport: l'ALPINISME a développé en France le goût des études géographiques, géologiques et météorologiques. L'ALPINISME vivifie.» Nouveau Larousse illustré. Remarquons qu'il semblerait plutôt que ce soit le goût pour la connaissance scientifique qui ait développé le goût pour l'alpinisme.

Le mot est absent du dictionnaire de Littré, qui, curieusement, enregistre alpiniste dans son Supplément. L'acteur était reconnu, non le concept. Il est vrai que nous n'avons rencontré le mot dans notre documentation qu'à partir de 1876 ( $c f$. Tetet 1985). En 1890, le Dictionnaire général l'ignore encore.

Alpinisme est enregistré pour la première fois dans un dictionnaire de langue, en 1953, dans le Robert: «Sport qui consiste à faire des ascensions, des excursions dans les Alpes, et par ext., dans n'importe quelle montagne.» L'alpinisme est reconnu comme discipline sportive.

À la fin du XIX ${ }^{\mathrm{e}}$ siècle, les nomenclatures alpines sont extrêmement réduites. Quelques mots : ascension, ascensionniste, bâton, course, guide, piolet, rimaye, qui appartiennent en fait au vocabulaire des guides, dont l'activité était bien connue depuis longtemps déjà : il s'agissait de faire passer les cols des Alpes à des fins utilitaires ou militaires et ce, dès le Moyen Âge. Or, 300 termes étaient déjà en usage dans le milieu alpin, dont certains depuis la fin du XVIII siècle, quand sont publiés les Voyages dans les Alpes d' H.B. de Saussure 10 .

Les raisons qui expliquent ces lacunes sont multiples : elles tiennent d'une part aux options du lexicographe : enregistrer les termes susceptibles d'être rencontrés par un public de lecteurs cultivés; et les termes en usage. Or l'ascension des montagnes, à visée scientifique, est une activité propre aux savants; la connaissance des ouvrages alors publiés reste limitée au milieu au sein duquel s'exerce l'activité. La littérature alpine, représentée par les récits de voyage - une cinquantaine recensés au XVIII siècle - n'appartient pas à un genre littéraire méritant d'être retenu. L'alpinisme ne fait l'objet d'aucun texte à caractère journalistique, qui aurait pu toucher un public plus vaste et assurer une certaine diffusion à ce vocabulaire. D'autre part, à la fin du XIX $\mathrm{X}^{\mathrm{e}}$ siècle, en France, le sport en général, considéré comme une activité pour oisifs et riches, n'est pas digne d'attention.

C'est la publication du dictionnaire de Paul Robert qui va traduire, sur le plan lexicographique, l'intérêt d'un public de non-spécialistes pour un sport qu'il découvre à travers un genre littéraire nouveau: la littérature alpine romanesque. Le roman de $R$. Frison-Roche, Premier de cordée, publié en 1941, connaît un immense succès. D'où la présence à la nomenclature de termes marqués courants: cairn, cordée, encorder ( $\mathbf{s}$ '), escalader, piton, prise, rappel, refuge, varappe ${ }^{11}$.

Durant cette période de la première moitié du $\mathrm{XX}^{\mathrm{e}}$ siècle, le milieu alpin a créé près d'un millier de termes, dont un tiers au moins d'un usage courant.

En 1970, lorsque paraît le Supplément au Dictionnaire Robert, le grand public a été fortement sensibilisé à l'exploit alpin après les victoires sur l'Annapurna et l'Everest. La presse et les radios en ont rendu compte. Des films ont été projetés; des conférences organisées. Le Supplément enregistre alors une trentaine de termes très marqués du vocabulaire de l'alpinisme, extrêmement courants, depuis plus de 30 ans, voire 100 ans, dans le milieu alpin : assurance, assurer, auto-assurance, baignoire, botter, cramponnage $[\ldots]$ dévisser, glaciairiste, pendule [...] ramonage, sherpa. Seul sherpa appartient à l'alpinisme d'expédition. Donc, malgré l'évolution qu'a connue l'alpinisme entre 1950 et 1970, sur le plan technique, technologique, en raison de ses nouveaux objectifs, il n'apparaît, dans le Supplément, aucun terme significatif de cette période. 
L'année 1970 voit la publication du premier dictionnaire spécialisé de l'alpinisme: le Dictionnaire de la montagne de Jacques Gautrat, dictionnaire qui, jusqu'en 1982, date de publication du Robert des sports, constituera, pour les lexicographes de dictionnaires généraux, le dictionnaire de référence. Dictionnaire de type encyclopédique, le dictionnaire de Gautrat enregistre quelques noms propres et des énoncés de nature extralinguistique. La nomenclature est constituée par 250 entrées lexicales. Gautrat enregistre les termes les plus marqués du vocabulaire de l'alpinisme, que le non-spécialiste n'est pas en mesure de décoder en faisant appel à son seul sentiment linguistique : bénitier, descendeur, gratton, jumar, opposition, pianoter, etc.

La richesse du dictionnaire de Gautrat n'en laisse pas moins apparaître de nombreuses lacunes, qui correspondent à des référents et concepts nouveaux apparus entre 1950 et 1970. L'alpinisme représenté dans Gautrat est celui de la première moitié du XX $\mathrm{XX}^{\mathrm{e}}$ siècle. Par conséquent, le dictionnaire de Gautrat constituant la principale source des dictionnaires généraux, ceux-ci élaborent une nomenclature de termes, certes en usage, mais qui demanderait à être complétée, actualisée, pour être représentative de la discipline, telle qu'elle se pratique aujourd'hui.

En 1982 Georges Petiot publie le Robert des sports. Un dictionnaire de la langue des sports, qui se fonde sur les dépouillements de textes spécialisés pour élaborer les définitions, fournir des exemples cités, proposer des dates de première attestation. La publication du Robert des sports correspondait à un besoin. Négligés par les lexicographes, les vocabulaires sportifs dans leur ensemble et leur diversité n'avaient jamais jusqu'alors retenu plus particulièrement leur attention.

Avec, pour l'alpinisme, 250 termes, le Robert des sports propose une nomenclature relativement riche élaborée en référence à une littérature spécialisée: récits de courses, ouvrages techniques, périodiques spécialisés; les plus anciens textes étant représentés par les ouvrages de H.B. de Saussure, le plus récent datant de 1978. Le Robert des sports n'enregistre pas les termes les moins marqués de l'alpinisme, qui figurent dans les dictionnaires généraux, ni les termes ultra spécialisés qui feraient plutôt l'objet d'un dictionnaire spécialisé. Il enregistre les termes d'un usage fréquent dans le milieu, susceptibles d'être rencontrés par un lectorat d'amateurs. C'est un ouvrage qui s'adresse aux non-spécialistes et qui a donc vocation à être exploité par les dictionnaires généraux.

Mais à l'analyse, il apparaît que le nombre de termes apparus entre 1970 et 1982 est infime. Dix ans de pratique alpine sont ignorés. Quant à l'escalade moderne sportive, elle est totalement occultée. Les lacunes sont nombreuses mais il ne pouvait en être autrement dans un ouvrage qui se voulait multi-sports, fabriqué de manière «artisanale». Il n'en reste pas moins qu'à ce jour, il constitue pour les dictionnaires généraux la source essentielle à laquelle ils se réfèrent pour traiter des vocabulaires sportifs.

Dans les grands dictionnaires de langue actuels, le Trésor de la langue française et Le Grand Robert, les nomenclatures alpines sont représentées, approximativement, par 200 termes. Les textes de littérature générale, quelques textes de littérature alpine de la première moitié du XX $\mathrm{XX}^{\mathrm{e}}$ siècle et les dictionnaires de Gautrat et Petiot constituent l'essentiel du corpus de travail. D'où l'absence de dénominations relatives à l'alpinisme actuel et à l'escalade en tant que discipline autonome. Les textes littéraires retenus comme représentatifs appartiennent pour la plupart à la littérature alpine romanesque. Il s'agit essentiellement de romans de Frison-Roche: Premier de cordée, La grande crevasse; de deux récits de courses: 342 heures dans les grandes Jorasses de R. Desmaison, des Conquérants de l'Inutile de L. Terray, et dans les dix premiers volumes du TLF, de Matterhorn, de J. Peyré, paru en 1939. Alors que plus d'une centaine de romans de montagne ont été recensés entre 1902 et 1950 (Ballerini 1973), deux seulement ont été retenus. Le premier, en raison 
de son succès; le second, pour la notoriété acquise par son auteur qui reçut le prix Goncourt pour un autre roman, et bien sûr, la qualité romanesque de l'ouvrage ${ }^{12}$.

Depuis 1950 environ, le roman de montagne n'est plus représentatif de la littérature alpine. Celle-ci s'exprime essentiellement dans les récits de courses, les topoguides, les ouvrages techniques. Et dans les périodiques. Jusqu'en 1979, les périodiques alpins écrits par des alpinistes pour les alpinistes, n'étaient diffusés que dans le milieu alpin. À partir de cette date, d'autres périodiques paraissent, qui sont mis à la disposition de tout public dans les Maisons de presse. Écrits par des journalistes-alpinistes, ils sont l'exacte représentation de l'actualité alpine dans les modalités de la pratique, l'utilisation de nouvelles techniques, de nouveaux matériaux et l'émergence d'une nouvelle éthique. Il n'est plus possible aujourd'hui au lexicographe qui travaille sur corpus d'opérer une sélection de textes en fonction de critères plus culturels que pertinents. Aucun texte littéraire, aussi recevable soit-il, n'est représentatif d'un vocabulaire de spécialité, quand celui-ci s'exprime à travers des textes qui lui sont propres. Le critère de diffusion, si souvent évoqué par le lexicographe pour justifier ses choix, reste pertinent, s'il ne se limite pas à une diffusion comprise à travers une certaine forme de littérature. Un inventaire exhaustif, actuel et varié devra désormais être établi en concertation avec les spécialistes du domaine avant d'entreprendre d'élaborer une nomenclature représentative de la discipline; ce qui se justifie d'autant plus qu'il n'existe à ce jour aucun dictionnaire spéçialisé récent dans ce domaine.

La nomenclature du dictionnaire multilingue spécialisé, lorsqu'il existe, est toujours plus complète. Le dictionnaire multilingue s'adresse à ceux qui ont besoin de connaître, dans l'exercice de leur activité, l'équivalent dans une langue étrangère des termes utilisés par la discipline. Il répond à un besoin immédiat. En 1989, paraît le Dictionnaire alpin en quatre langues: français, allemand, anglais, italien, élaboré par des alpinistes germanophones. Un dictionnaire dont le but «aider à comprendre les descriptions des guides dans une autre langue, à pouvoir lire les revues et livres spécialisés dans une autre langue, à faciliter les entretiens et discussions, non seulement au cours de sessions et de congrès, mais également dans les refuges et au sommet des montagnes» («Préface», p. 229), traduit bien les préoccupations du monde alpin face à une inflation lexicale qui constitue un obstacle à la communication entre les protagonistes d'une activité à dimension internationale. Avec l'ouverture des frontières et la mise en place de l'«Europe des guides» tout guide membre de la Communauté européenne, ayant reçu une formation conforme à la plateforme communautaire, aurait désormais le droit d'exercer son activité en tant que professionnel avec des clients français, sur les montagnes françaises, et réciproquement - un répertoire multilingue des termes de l'alpinisme devient un outil de travail au sein d'une profession qui va être confrontée à la dure loi de la concurrence. On peut facilement imaginer qu'à compétences égales, le client choisira son guide en fonction des facilités qu'il aura à communiquer avec lui.

Comme tout lexique élaboré par des spécialistes du domaine, sans l'aide des spécialistes des mots, le Dictionnaire alpin n'est pas exempt des défauts inhérents à ce type d'ouvrages. Sur les 2600 entrées répertoriées, un peu plus du tiers seulement peut être considéré comme appartenant au vocabulaire alpin, quand on a exclu de la nomenclature les mots du vocabulaire général et des énoncés phraséologiques, qui correspondent à la traduction d'une unité lexicale en allemand.

Pour la première fois un répertoire de type dictionnairique enregistre des termes représentatifs de l'alpinisme actuel: alpinisme extrême, escalade extrême, de ses moyens: baudrier, broche à glace, corde bicolore, corde multi-chutes, crampons articulés, piton à glace, etc., de ses techniques : cramponnage frontal, etc. Mais les lacunes restent nombreuses: des concepts aussi usuels que le à-vue, l'enchaînemient, la moulinette, le 
travail (des voies) sont absents; absentes également les dénominations de matériels et équipements courants : chausson d'escalade, dégaine, magnésie, etc. La nomenclature reste hybride, hésitant entre le mot-concept et le mot-unité lexicale; figurent ainsi à la nomenclature: grimper jusqu'à la limite de ses possibilités, grimpeur expérimenté, grimpeur solitaire - grimpeur solitaire étant par ailleurs le seul élément lexicalisé sur les trois groupements cités - alors que grimpe, grimpeur et grimper sont absents. Le Dictionnaire alpin enregistre en grand nombre, ce qui, dans les dictionnaires de langue monolingues, constituerait les exemples d'emploi des termes enregistrés : des syntagmes: broche à épines, broche à glace semi-tubulaire, broche tire-bouchon ; coinceur biconique ; coinceur gigogne, etc.; et des locutions : avaler la corde, enrouler, plier la corde, récupérer la corde, etc. D'où le succès rencontré par cet ouvrage auprès des professionnels de la montagne.

Nous n'insisterons pas plus sur l'intérêt d'un tel répertoire à l'usage des spécialistes ${ }^{13}$.

Il n'est pas envisageable, bien évidemment, qu'un dictionnaire général, inclue dans sa nomenclature l'ensemble des termes en usage dans un milieu de spécialistes. Il n'en reste pas moins que le dictionnaire général doit tendre à une représentativité aussi précise et exacte que possible de la réalité lexicale des domaines considérés. À défaut de disposer du dictionnaire spécialisé, le dictionnaire multilingue peut aider à approcher cette réalité. Il est une source d'information non négligeable qu'il serait bon que le lexicographe de dictionnaire de langue prenne en compte ${ }^{14}$.

En introduction à cet article, nous avons précisé que nous ne traiterions pas des problèmes posés par la définition des unités spécialisées, et leur exemplification. Ces problèmes sont toujours largement évoqués et débattus dans les revues et lors de rencontres. Les problèmes sont les mêmes, que l'on traite des vocabulaires scientifiques, techniques ou sportifs.

Par contre, et c'est sur quoi nous voudrions maintenant insister, il est rarement question des informations historiques concernant les termes de spécialités, à travers, plus particulièrement, les propositions de première attestation, qui entrent dans le programme lexicographique des dictionnaires de langue.

\section{L'INFORMATION HISTORIQUE}

C'est aujourd'hui dans les dictionnaires que se fait l'histoire de la langue 15 : soit que le dictionnaire inclue dans son programme les informations historiques sur le mot (TLF, Grand Larousse de la langue française, Grand Robert, Petit Robert), soit que l'histoire des mots constitue le seul programme d'un ouvrage: la publication, fin 1992, du Robert historique en est le meilleur exemple.

«Parmi les différents éléments d'information que la lexicographie a la charge d'inventorier, l'un des plus significatifs est représenté par les premières attestations ou datations des mots, entourés des données graphiques, phonétiques, morphologiques, sémantiques, stylistiques, etc. qu'elles apportent avec leur contexte.» Lorsqu'en 1959, Bernard Quemada préface ainsi le premier volume des Matériaux pour l' histoire du vocabulaire français, il va donner à la recherche historique une dimension qui se traduira, pendant plus de 30 ans, par la publication de Datations nouvelles ${ }^{16}$, largement reprises au fil des années par les dictionnaires à visée historique. La mise à disposition des lexicographes de ces données prédictionnairiques constitue pour l'information historique un élément déterminant de sa crédibilité. La méfiance des curieux de l'histoire des mots quant à la valeur des datations proposées par les lexicographes: «nous sommes de plus en plus persuadé que, pour une grande partie du vocabulaire, les indications historiques des dictionnaires ne répondent ni au point de vue philologique ni au point de vue linguistique, aux exi- 
gences de notre temps» (Höfler 1986: 243), «Toutes les datations à 0,01\% près sont fausses...» (Enckell 1987: 34), a, il est vrai, pendant longtemps, été justifiée.

La recherche systématique que nous avons entreprise des premières attestations des termes d'alpinisme, confirmerait, s'il en était besoin, le caractère tout relatif des datations proposées dans les dictionnaires. À quelques termes près, nous avons pu reculer toutes les datations proposées, dans le domaine qui nous intéresse ici.

Ces modifications que nous avons pu apporter tiennent à la nature du corpus que nous avons retenu comme source de créativité lexicale. Le choix du corpus est déterminant dans la recherche des premières attestations des termes des vocabulaires de spécialités. Ce corpus ne peut, en aucun cas, être le même que celui qui est exploité par les lexicographes pour élaborer les définitions et fournir les citations qui entrent dans l'article du mot traité. Or il apparaît que dans la plupart des cas, un seul et même corpus a été exploité à des fins diverses, voire opposées.

Dans le $T L F$, la littérature générale et la littérature alpine romanesque sont à l'origine des datations proposées pour escalade (1816, Maine de Biran), se dérocher (1941, Frison-Roche), encordement (1939, Peyré), grimpeur (1855, Daudet); dans le GLLF, premier de cordée, prise, ramasse, ramoner, rappeler sont présentés comme attestés pour la première fois en 1941, date qui correspond à la publication du roman de FrisonRoche, Premier de cordée. Dans tous ces cas, et dans bien d'autres, il apparait que la datation proposée correspond à la date d'entrée du terme dans le milieu des non-spécialistes, à travers une littérature qui leur est destinée. Nous avons affaire à une datation-diffusion et non à une datation-création.

Ce type de datation-diffusion peut, ce qui nous semble plus grave, être de nature référentielle et non lexicale. L'événement crée alors la datation. Un seul exemple suffira à illustrer notre propos: Le Grand Robert date de 1950 la première attestation de sherpa; c'est en 1950, avec le succès de l'expédition française à l'Annapurna, que le mot se diffuse dans le public. Or les expéditions en Himalaya avaient débuté dès 1930; dès cette époque, le peuple Sherpa fournissait des porteurs. Sherpa «porteur» est attesté dès 1933 et très souvent avant 1950. La datation proposée correspond donc ici à la datation d'un événement - l'ascension de l'Annapurna - et non à la datation du mot.

L'utilisation de dictionnaires comme sources de datations - «En dépit de dépouillements considérables de textes, les dictionnaires sont restés à présent un des principaux moyens de datation des mots français» (Höfler 1982: 292) - confirme cette assertion: ascension : 1845-46, Bescherelle (TLF); ascensionniste : Littré (RSp.); cheminée : 1890, Dictionnaire général (TLF); cordée: 1907, Larousse (TLF); cramponnage : 1961, Larousse encyclopédique (TLF), mousqueton: 1963, Larousse encyclopédique (TLF). Dans le Robert des sports, c'est surtout le dictionnaire de Gautrat qui est source de datations, pour une trentaine de termes : antécime, avaler le mou, baquet, bénitier, etc. ${ }^{17}$. À partir du moment où un terme fait l'objet d'une entrée dans un dictionnaire, général ou spécialisé, on peut être assuré qu'il est entré dans la langue, générale ou dans celle des spécialistes, depuis longtemps déjà. Dans ce cas, nous avons là encore affaire à une datation-diffusion. Datation à laquelle le consultant initié n'accordera qu'une valeur toute relative, mais que le consultant peu enclin à mettre en doute la vérité du dictionnaire considérera comme incontestable. Ce type de datation ne peut être qu'un repère dans la recherche de la véritable datation qui est l'attestation première d'une forme ou d'un emploi dans le milieu des spécialistes.

Le Robert des sports, en exploitant un corpus de textes spécialisés constitué de récits de courses et de manuels techniques pour l'essentiel, est plus proche de la réalité lexicale historique. Les datations du Robert des sports sont souvent très antérieures à celles proposées par les dictionnaires généraux : ancrage : $T L F, 1967 ; R S p ., 1937$; ascension : $T L F, 1845$ - 
46; RSp., 1787 ; attaque : $T L F, 1970 ; R S p ., 1955$; cordée : $T L F, 1907$; RSp., 1886; etc. Elles ne constituent pourtant qu'une étape intermédiaire entre le moment où le terme a véritablement été créé et le moment, évoqué ci-dessus, où il est apparu dans le public des non-spécialistes. Les datations du Robert des sports marquent l'entrée du terme comme terme en usage dans le milieu des spécialistes: les récits de course et les manuels techniques qui ne sont pas novateurs en matière de vocabulaire, entérinent un vocabulaire déjà largement diffusé.

La véritable datation, celle qui résulte d'une volonté de création en raison de l'apparition de nouveaux référents dans une réalité extra-linguistique mouvante, est à chercher ailleurs. Dans la discipline qui nous intéresse ici, les périodiques constituent la source essentielle du repérage des premières attestations des termes alpins. L'alpiniste aime se raconter et faire partager aux autres ses émotions et ses découvertes. D'où, dès 1874, la publication de périodiques par le Club alpin : périodiques issus du Club à l'échelon national et périodiques produits par ses sections. C'est le dépouillement de ces périodiques qui nous a permis de proposer des datations que nous pouvons considérer comme étant les plus proches de la date de création des termes en question; dans ce domaine de la recherche, la modestie nous oblige à rester prudente et à ne jamais considérer comme définitive une datation que la découverte d'un nouveau texte pourra remettre en question. Mais le paysage dictionnairique alpin, dans sa composante historique, s'en trouve considérablement modifié.

Nous avons ainsi pu proposer des attestations antérieures (Tetet 1985) à celles proposées par le $R S p$., parfois de plus d'un siècle, le plus souvent d'au moins une trentaine d'années, voire une cinquantaine : assurage: $R S p$., 1970; $D D L, 1932$; attaquer: $R S p ., 1868 ; D D L, 1786$; escalader : $R S p ., 1804 ; D D L, 1886$; face : $R S p ., 1937 ; D D L$, 1773 ; itinéraire : $R S p ., 1940 ; D D L, 1897$; marche d'approche: $R S p ., 1937 ; D D L$, 1909 ; penduler : $R S p$., $1941 ; D D L, 1912$, etc.

Suivant les ouvrages consultés, l'information historique s'avère donc extrêmement disparate : soit que la première attestation d'un terme corresponde à la date d'entrée de ce terme dans le milieu des non-spécialistes - c'est le cas dans la plupart des dictionnaires de langue généraux - soit qu'elle corresponde à la date de diffusion du terme dans le milieu des spécialistes; ce qui est le cas dans le dictionnaire de langue spécialisé. En fait, nous avons affaire à des dates d'attestation des termes à différents moments de leur vie lexicale. Ce qui n'apparaît pas toujours clairement dans les propos des lexicographes en préface à leurs ouvrages et peut laisser penser au consultant que l'attestation proposée est datation du terme. Ce n'est peut-être qu'une question de terminologie qu'il serait souhaitable de préciser.

\section{CONCLUSION}

À partir d'un corpus de recherche constitué par un ensemble de termes appartenant au vocabulaire de l'alpinisme, nous avons mis en évidence les faiblesses du traitement lexicographique: nomenclature insuffisante, périmée parfois, inactuelle toujours; information historique pour le moins discutable.

L'ignorance de la réalité extra-linguistique, l'inadéquation des corpus à cette même réalité sont source de lacunes et d'imprécisions qui portent préjudice à la discipline dans sa représentativité lexicale, et par conséquent à la langue, dont elle est une des manifestations.

Quant à l'étude historique des vocabulaires de spécialités, elle est à reconsidérer dans son ensemble: à partir du moment où un terme est affaire de spécialistes, il ne peut, historiquement, être envisagé hors du milieu qui l'a créé. Dans cette perspective historique, seul le texte spécialisé, écrit par le spécialiste à l'usagé du spécialiste, est pertinent. 
Ce qui signifie des corpus de travail différents suivant que le programme lexicographique envisage ces vocabulaires dans leurs représentations synchronique ou diachronique.

Nous pouvons, sans trop nous tromper, avancer que le traitement lexicographique de tous les vocabulaires de spécialités est à revoir, que les ouvrages s'adressent aux spécialistes de la langue ou aux spécialistes du domaine ou de la discipline. Un grand chantier en perspective qui devrait faire les beaux jours des lexicographes de demain.

\section{Notes}

1. Cf. Callebaut (1983), Candel (1979; 1983), Mazière (1981), etc.

2. Pour une étude critique de l'ensemble des informations, voir Tetet (1991), tome I.

3. Dans le sens où on l'entend habituellement. Mais la compétition était sous-jacente entre alpinistes de différentes nationalités, qui tentaient d'atteindre un sommet pour la gloire de leur pays.

4. Sur les 1300 guides syndiqués. Chiffres fournis par le Syndicat national des guides (Chamonix).

5. Chiffre fourni par D. Taupin, auteur du Guide des sites naturels d'escalade en France, Paris, FFMECOSIROC, en cours de réédition.

6. Dans le milieu alpin, la dénomination «escalade à mains nues», irrite; elle n'a de signification que pour un public non averti qui comprend sous ce terme une forme d'escalade extrêmement rare - qui ne fait appel à aucun matériel d'assurance - fortement médiatisée.

Grimpe est considéré dans ce même dictionnaire comme un mot d'origine régionale, une information pour le moins étonnante. Grimpe est un déverbal formé sur grimper.

7. Bourdeau, Ph. (1990) : «L'escalade», Escalade, Actes du colloque Chamonix 89, pp. 83-88.

8. Répertoriés dans les Datations et documents lexicographiques, $\mathrm{n}^{\circ} 27$ et dans les Mots nouveaux contemporains, $n^{\circ} 2$, sous presse.

9. À paraître dans un lexique multilingue de l'alpinisme, en préparation.

10. Saussure (H.B. de), Voyages dans les Alpes, Genève-Neuchâtel, 1779-1796.

11. De nombreux termes présents dans le roman sont omis, bien que d'un usage courant: assurer, bivouaquer, caravane, corde de rappel, dalle, etc.

12. Précisons que J. Peyré n'était pas alpiniste et que les mots alpinisme et alpiniste n'apparaissent jamais dans l'ouvrage.

13. À la demande des professionnels de la montagne, à travers leurs institutions: Union internationale des Associations d'alpinisme, Fédération française de la montagne et de l'escalade, École nationale de Ski et d'Alpinisme, j'ai été chargée de mettre au point un dictionnaire multilingue - français, anglais, allemand, italien, espagnol - qui devrait permettre de combler les lacunes du Dictionnaire alpin.

14. Une autre source d'information de nature lexicographique est constituée par les glossaires qui figurent dans les ouvrages techniques. Ceux-ci reflètent fidèlement l'évolution de la discipline. Ainsi ce sont les seuls à gloser : autobloquant, à-vue, bidoigt, coincement, continuité, couenne, dégaine, exposé, fiffi, flash, main courante, monodoigt, moulinette, pof, spit, voler, etc.

15. Les années 1950-1960 ont été riches en travaux de lexicologie historique, menés par Arveiller, Dubois, Guiraud, Guilbert, Matoré, Quemada, Wexler. Aujourd'hui la continuation de l'Histoire de la langue française semble traduire un nouvel intérêt pour ce type d'approche.

16. Datations et Documents Lexicographiques (DDL), 41 volumes parus de 1970 à 1993, CNRS, INaLF, Diffusion Klincksieck.

17. Dans le Robert des sports, la citation, outre la fonction qui lui est propre, étant mise au service de la définition et de l'attestation historique, il s'en suit une certaine confusion dans les informations.

\section{RÉFÉRENCES}

Dictionnaires et lexiques

Dictionnaire alpin français-allemand-anglais-italien (1989) : avec la collaboration de Hélène Collonge pour la partie française, Munich, Éd. Rudolf Rother.

GAUTRAT, J. (1970) : Dictionnaire de la montagne, Paris, Éd. du Seuil, Coll. «Microcosme».

HATZFELD, A., DARMESTETER, A. et A. THOMAS (1890-1900): Dictionnaire général de la langue française, Delagrave.

LAROUSSE, P. (1866-1890): Grand dictionnaire universel du XIXe siècle, français, historique, géographique, biographique, etc., 15 vol. et 2 suppléments, Paris.

Larousse illustré (Le Petit) (1993) : Paris, Larousse.

LITTRÉ, É. (1863-1877) : Dictionnaire de la langue française, Paris, Hachette.

PETIOT, G. (1982) : Le Robert des sports. Dictionnaire de la langue des sports, Paris, Le Robert, [RSp.].

ROBERT, P. (1953-1964) : Dictionnaire alphabétique et analogique de la langue française, 6 vol., Paris, Société du Nouveau Littré. 
ROBERT, P. (1985): Le Grand Robert de la langue française. Dictionnaire alphabétique et analogique de la langue française, 9 vol., $2^{e}$ édition, entièrement revue et enrichie par A. Rey, Paris, Le Robert.

Robert (Le Nouveau Petit) (1993) : sous la direction de J. Rey-Debove et A. Rey, nouvelle édition remaniée et amplifiée, Paris, Le Robert.

TETET, Ch. (1985) : Datations et Documents Lexicographiques. Matériaux pour l' histoire du vocabulaire français. Les sports de montagne, $\mathrm{n}^{\circ} 27$, L'alpinisme.I (1757-1975), CNRS, INaLF, Diff. Klincksieck, [DDL].

Trésor de la langue française. Dictionnaire de la langue du 19e et du 20e siècle (1978-1992): sous la direction de P. Imbs, puis de B. Quemada, Éd. du CNRS, Gallimard, 15 volumes parus [TLF].

\section{Ouvrages et revues}

BALLERINI, M. (1973) : Le Roman de montagne en France, Arthaud, Coll. Sempervivum.

CALLEBAUT, B. (1983): «Les vocabulaires techniques et scientifiques et les dictionnaires», Cahiers de lexicologie, 43 , pp. 33-52.

CANDEL, D. (1979) : «La présentation par domaines des emplois scientifiques et techniques dans quelques dictionnaires de langue», Langue française, 43 , pp. 100-115.

CANDEL, D. (1983): «Réflexions sur l'utilisation de textes scientifiques dans un dictionnaire de langue», Études de linguistiques appliquée, 51, pp. 21-33.

ENCKELL, P. (1987) : propos recueillis par Ibrahim, A. : «Un chercheur qui n'arrête pas de trouver», Diagonales, supplément au n ${ }^{\circ} 210$ du Français dans le monde, pp. 34-35.

HÖFLER, M. (1986) : «Typologie des erreurs de datation dans la lexicographie française», Revue de linguistique romane, 50 , pp. $423-442$.

MAZIËRE, F. (1981) : «Le dictionnaire et les termes», Cahiers de lexicologie, 39, pp. 79-104.

TETET, Ch. (1988) : «Réflexions autour de la lexicographie alpine. État présent de la question et perspectives», La Montagne et alpinisme, 154, p. 73.

TETET, Ch. (1990): «Approche lexicologique et lexicographique dans une perspective historique et multilingue du vocabulaire du grimpeur», Escalade, Actes du colloque Chamonix 89, pp. 30-39.

TETET, Ch. (1991): Contribution au traitement lexicographique des vocabulaires spécialisés. Le concept de diffusion dans le traitement lexicographique du vocabulaire de l'alpinisme, Thèse de Doctorat, 2 tomes, Université de Franche-Comté, 1991. 